to care, reduce waiting times and NHS costs (Department of Health, 2014).

One example of this collaboration is the Video Fluoroscopy Swallow Service provided by Radiographers and Speech and Language Therapists (SLT) at Great Ormond Street Hospital. The service provides accurate, low dose x-ray screening for the assessment of swallow physiology in children. Results are used to inform multi-disciplinary management of eating and drinking difficulties, including aspiration risk. We outline the service and explain how this method of collaborative working harnesses the clinical expertise of both professions, providing a high-quality service which contributes to the improvement of patient outcomes and experience.

Learning outcomes from this service include the importance of having clearly defined AHP roles, and patient suitability criteria as well as excellent communication between the whole multi-disciplinary team. The opportunity for extended scope practice provides staff with job satisfaction and skill development. This Video Fluoroscopy swallow study model highlights how AHP's working collaboratively can make a vital contribution to efficient use of clinical spaces, reducing costs and waiting lists while providing high quality care for children at Great Ormond Street Hospital.

It is essential that such collaborations at Great Ormond Street Hospital are celebrated and actively encouraged enabling service improvements.

\section{CONTROLLED DRUGS STORAGE AND IMPROVEMENT}

${ }^{1}$ Andrew Pearson, ${ }^{2}$ Hong Thoong, ${ }^{2}$ Rasha Shamsah, ${ }^{2}$ Ka Yu Yung. ${ }^{1}$ Great Ormond Street Hospital; ${ }^{2} \mathrm{GOSH}$

\subsection{6/archdischild-2020-gosh.81}

Learning from a Serious Incident in 2018 highlighted the importance of the documentation of controlled drugs. A detailed audit was conducted in July 2019. The main areas for improvement across the trust were around documentation in the $\mathrm{CD}$ order book and $\mathrm{CD}$ register. An action plan was agreed between nursing education and pharmacy departments to support best practice. This included the development of digestible best practice guidance to be displayed in medicine storage rooms, revision of policy and education roll out which took take place in September 2019 Changes have been made and through the audit process we have improved our standards.

$\%$ of all standards met

Jul 2019 80\%

December 2019 85\%

August 2020 90\%

Feeding back audit results quickly and visually has been useful. Thankyou to all pharmacists and ward staff who have supported this work. We have made changes and reduced risk in this area.

\section{EVALUATION OF THE MEDICINES MATTER NEWSLETTER IN GOSH}

Ka Yu Yung. Great Ormond Street Hospital

10.1136/archdischild-2020-gosh.82
Medicines form the largest group of interventions used in children and young people. It also accounts for the highest number of clinical errors. A newsletter 'Medicines Matter' designed to support good use of medicines within the Trust was introduced in October 2019 and published monthly by Pharmacy. The newsletter, intended to be read by all healthcare professionals, highlights issues and provides updates on all aspects of medicines management. The contents of the newsletter includes incident examples, along with tips in mitigation, new drugs approved by the Drugs \& Therapeutic Committee, new resources, MHRA drug safety update, drug shortages, practice and governance issues.

A feedback survey was conducted to evaluate its' effectiveness as a communication forum as well as staff's views of it. A survey with 9 questions was circulated with the July 2020 edition and answers were collected until September 2020. Sixteen staff members completed the survey where nursing staff dominated (69\%), followed by allied health professionals (25\%) and medical staff (6\%). $80 \%$ of participants read every or most issues with the remaining reading some issues. All participants rated the newsletter as good or very good and useful or very useful where at least $60 \%$ of the respondents were interested in each of the topics within the newsletter. The content was found to be engaging (53\%), educational (80\%), informative (100\%), fun-to-read (33\%) and a quick reference to changes that are happening $(7 \%)$. A larger percentage $(60 \%)$ of participants did not know where to find the newsletter and a suggestion were made to include some medication-related success stories and addition of hyperlinks to new resources available. One participant found the layout and format slightly disorientating but the overall response was positive. The feedback suggests that the newsletter provides a good platform to deliver up-to-date information and key communication across the organisation.

\section{A MULTIVARIABLE COMPOSITE OUTCOME TO DEFINE DISEASE SEVERITY IN CHILDREN WITH CYSTIC FIBROSIS}

${ }^{1}$ Nicole Filipow, ${ }^{1}$ Gwyneth Davies, ${ }^{1}$ Eleanor Main, ${ }^{2}$ Sanja Stanojevic. ${ }^{1}$ Great Ormond Street Hospital and University College London/ICH BRC; ${ }^{2}$ Dalhousie University

\subsection{6/archdischild-2020-gosh.83}

Introduction Improvements in cystic fibrosis (CF) care have resulted in improved outcomes, specifically many children maintain lung function in the normal range. Nonetheless there are children with poor outcomes, and there is a need for a more comprehensive multi-factorial measure that summarises the overall health status in this new era of CF care.

Objective To define phenotypically distinct clusters of pediatric CF patients that are linked to different health outcomes by using basic machine learning algorithms.

Method Data from the Toronto CF Clinical Database were used to define phenotypic clusters based on a broad variety of patient-descriptive variables. A Partitioning Around Medoids (PAM) clustering method was iteratively carried out on different combinations of the variables until a maximum distinction between outcome measures could be identified, which included time to recurrent event analyses for both pulmonary exacerbations and hospital admissions. The results were validated in GOSH CF clinical data housed within the GOSH-DRIVE DRE. 